\title{
RAMSEY'S THEORY MEETS THE HUMAN BRAIN CONNECTOME
}

\author{
Arturo Tozzi \\ Center for Nonlinear Science, Department of Physics, University of North Texas, Denton, Texas, USA \\ 1155 Union Circle, \#311427Denton, TX 76203-5017 USA \\ tozziarturo@libero.it
}

\begin{abstract}
Ramsey's theory (RAM) from combinatorics and network theory goes looking for regularities and repeated patterns inside structures equipped with nodes and edges. RAM represents the outcome of a dual methodological commitment: by one side a top-down approach evaluates the possible arrangement of specific subgraphs when the number of graph's vertices is already known, by another side a bottom-up approach calculates the possible number of graph's vertices when the arrangement of specific subgraphs is already known. Since natural neural networks are often represented in terms of graphs, we suggest to utilize RAM for the analytical and computational assessment of a peculiar structure supplied with neuronal vertices and axonal edges, i.e., the human brain connectome. We discuss how a RAM approach in neuroscientific issues might be able to locate and trace unexplored motifs shared between different cortical and subcortical subareas. Furthermore, we will describe how notable RAM outcomes, such as the Ramsey's theorem and the Ramsey's number, could contribute to uncover still unknown anatomical connexions endowed in neuronal networks and unexpected functional interactions among grey zones of the human brain.
\end{abstract}

Keywords: Computer Science, Machine Learning, extremal set theory; Turán's theorem, Ramsey’s theorem.

\section{INTRODUCTION}

Graphs are discrete mathematical structures equipped with vertices joined by edges (Wilson 2010; Diestel 2018). They represent the common, general manifolds subtending the phase spaces of our logical, mathematical, physical, chemical, biophysical and biological worlds. The Ramsey's theory (RAM) from combinatorics and network theory examines the intrinsic mathematical features endowed in graphs (Graham et al., 1990). Suggesting the general conditions for the existence of orderly substructures with regular properties, RAM advocates that large sets of elements (say numbers, points, planes, objects, image tessellations and so on) unavoidably contain repetitive geometric patterns (Bondy and Murty, 2008). Problems in RAM are usually tackled in terms of edge-coloured graphs such as, e.g., no matter how you $n$-color the edges, when the graph is large enough you will always find certain subgraphs with monochromatic edge labelling. A typical example of RAM is the problem of friends and strangers, which proves that at a gathering of any six people some three of them are either mutual acquaintances or complete strangers to each other.

By an operational standpoint, the aim of Ramsey's theory is to answer to one of the two following reversed questions:

1) Given a certain graph with $n$ vertices, are subgraphs with specific features present? In this case, researchers look for the unknown number of subgraphs encompassed in a graph with an already known number $n$ of vertices.

2) Given several subgraphs with specific features, which is the number $n$ of vertices that a certain graph must have to encompass them? In this reverse case, RAM looks for the required graphs with sufficiently many edges to contain already known structures as subgraphs. RAM counts how many elements of some structure must there be to guarantee the existence of a particular property.

RAM has been profitably used in far-flung scientific fields, since it concerns the emergence of order that occurs when every structure grows large enough. Interesting applications include game theory, decision making, metamathematics, communications, approximation algorithms, harmonic analysis, ergodic theory, convex and computational geometry, probabilistic method versus constructions, information retrieval in computer science, dual source codes, MaxSAT NPhard computation problems, lower bound arguments for Parallel Random-Access Machine and for Boolean function computation (Roberts 1984; Rosta, 2004; Molnár et al., 2018). 
Here we suggest to use RAM, well-suited for the evaluation of graphs, for the assessment of the anatomical/functional neural network known as the human brain connectome. Indeed, the cortical and subcortical area of the central nervous system form a hierarchical network characterized by community structures of densely interconnected brain subareas, each one standing for a graph vertex (Sporns et al., 2005; Reese et al., 2012; Peters et al., 2017). These networks are described at different coarse-grained scales, from single neurons to neuronal populations and nervous tissue's macroscale, corresponding to the various levels of spatial resolution of the currently available imaging techniques. Although the nervous networks' graphs are often too large to be manually analysed, advanced methods such as graph database and analysis libraries have been combined to search for and identify interesting subgraph patterns and motifs inside the ever-increasing amount of connectomics datasets (Matelsky et al., 2021).

Given these premises, we will at first provide a gentle introduction to RAM and its theorems, then we will describe how RAM can be used to look for unknown, orderly substructures with regular properties hidden in the human connectome.

\section{A THEOREM FROM RAMSEY'S THEORY}

Here we will focus on the Ramsey's theorem (RT) and the Ramsey's number, two of the most distinguished RAM outcomes. RT refers to complete graphs $\mathrm{K}_{n}$, i.e., graphs in which every pair of distinct vertices $n$ is connected by a unique undirected edge. To provide an example, Figure 1A illustrates a $\mathrm{K}_{6}$ complete graph. RT states that a complete monochromatic sub graph will always be found in any edge color labelling of a large enough complete graph (Graham and Rothschild, 1971). To provide an example, let the edges of $\mathrm{K}_{6}$ be coloured with two colours, e.g., black and grey, representing two different features (Figure 1B). If two vertexes are connected by, say, a black edge, this means that they both display the black feature. According to RT, no matter how you colour with black or grey the edges of $\mathrm{K}_{6}$, you cannot avoid either a $k$ triangle all of whose three sides are black, or a $l$ triangle all of whose three sides are grey (Figure 1B). The conclusion is straightforward: whatever colours are used, there will always be at least one monochromatic figure (that is, a figure with all the edges of the same color). Since $n$ vertices correspond to about $n 2$ edges, there are many ways to color the edges.

Therefore, for each pair of positive integers $k$ and $l$ there exists an integer $\mathrm{R}(k, l)$ (known as the Ramsey number) such that any graph with $\mathrm{R}(k, l)$ nodes contains a clique with at least $k$ nodes or an independent set with at least $l$ nodes. The general formula of the Ramsey's number reads:

$\mathrm{R}(k, l)=\mathrm{V}$

Where $k$ stands for the number of sides of the figure coloured with one color, $l$ stands for the number of sides of the figure coloured with another color, $\mathrm{R}(k, l)$ is an integer depending on both $\mathrm{r}$ and $\mathrm{s}$, and $\mathrm{V}$ stands for the number of vertexes (Allen 2008). The two terms $(k, l)$ suggest that we are using a 2-coloring of the edges. In other words, the Ramsey's number is the minimum number of vertices $v=\mathrm{R}(k, l)$ such that all undirected simple graphs of order $v$ contain a clique of order $k$ or an independent set of order $l$. RT states that such a number exists for all $k$ and $l$. Another statement of RT sounds as follows: for integers $k, l>=2$, there exists at least a positive integer $\mathrm{R}(k, l)$ such that no matter how the complete graph $\mathrm{K}_{\mathrm{R}(k, l)}$ is two-colored, it will contain a subgraph $\mathrm{K}_{k}$ or a subgraph $\mathrm{K}_{l}$.

The above-mentioned example of $\mathrm{K}_{6}$ is conventionally described by the formula:

$\mathrm{R}(3,3)=6$

The formula reads as follows: if you have a complete graph with the edges forming either a tree-sided black figure or a tree-sided grey figure, the complete graph must display at least 6 vertexes. In case of a subset of at least three completely uniform edges, RT guarantees that such subset is provided by a hexagonal graph.

RT copes with the existence of monochromatic subsets characterized by connected edges of just one colour. Nevertheless, when the number $n$ of the complete graphs' vertices increases, the number of possible colorings becomes very intricate. The Ramsey's numbers for the smallest $\mathrm{K}_{n}$ are known: for example, $\mathrm{R}(3,3)=6$ and $\mathrm{R}(4,4)=18$. However, when considering the huge available number of subgraphs with different colors, the Ramsey's number becomes almost impossible to calculate (Ajtai et al., 1980). For the higher $\mathrm{K}_{n}$, only quite poor bounds of the Ramsey's number are known (Angeltveit and McKay, 2017): for example R(5,5) is only known to lie in the range 43-49 (Figure 1C), while $\mathrm{R}(6,6)=102-165$.

RT works also for multidimensional graphs (Hindman and Strauss, 1998). The example of a hypercube is provided in Figure 1D. In this case, RT allows us to answer to the following questions:

a) What is the smallest number of dimensions of a $n$-dimensional hypercube, in which all vertex pairs form lines that all are colored with one of two colors (say black or grey), such that there must exist a monochromatic complete graph of four coplanar vertices? The answer is four.

b) What is the smallest number of edges of a hypercube such that all the available colorings will contain a monochromatic $\mathrm{K} 4$ with four coplanar vertices? The answer is eight. 
A

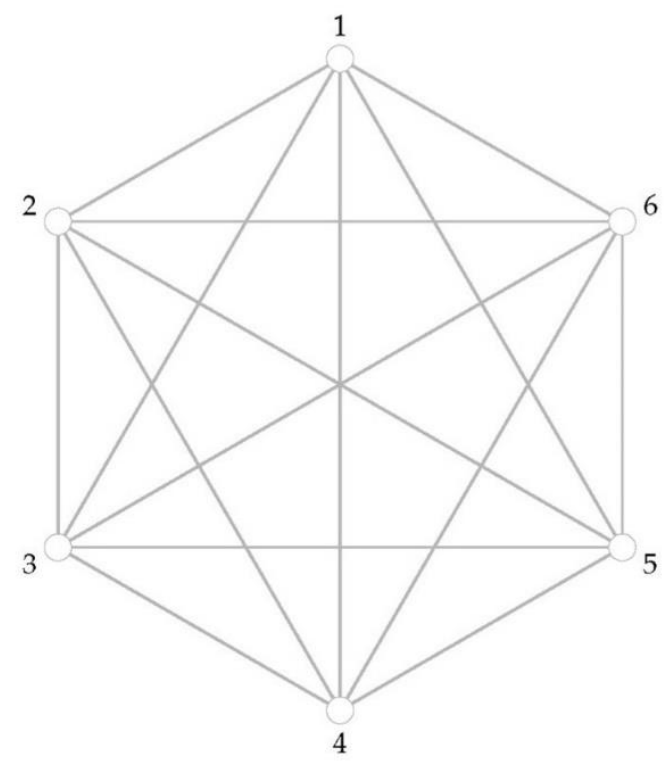

C

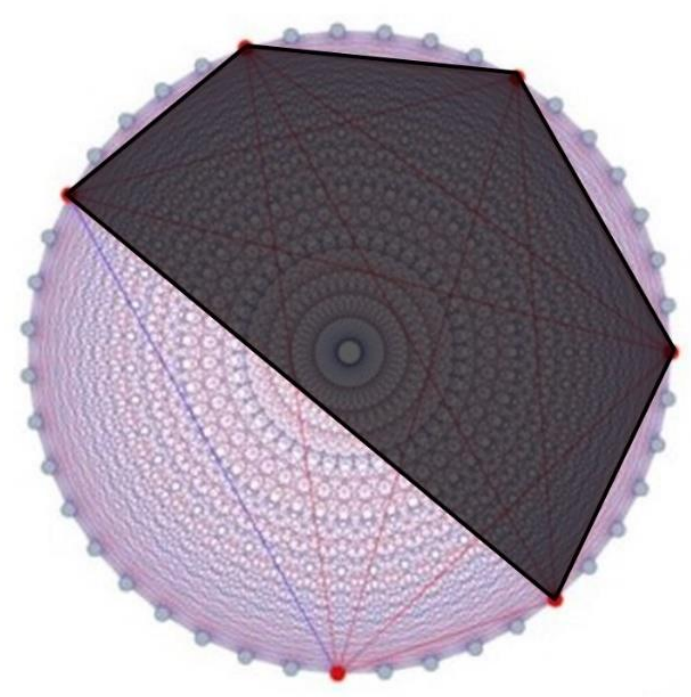

B

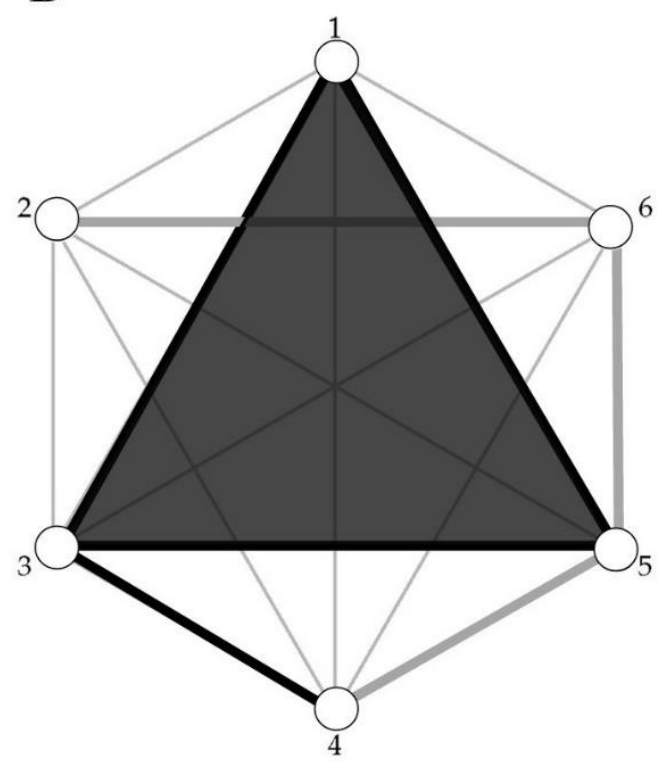

D

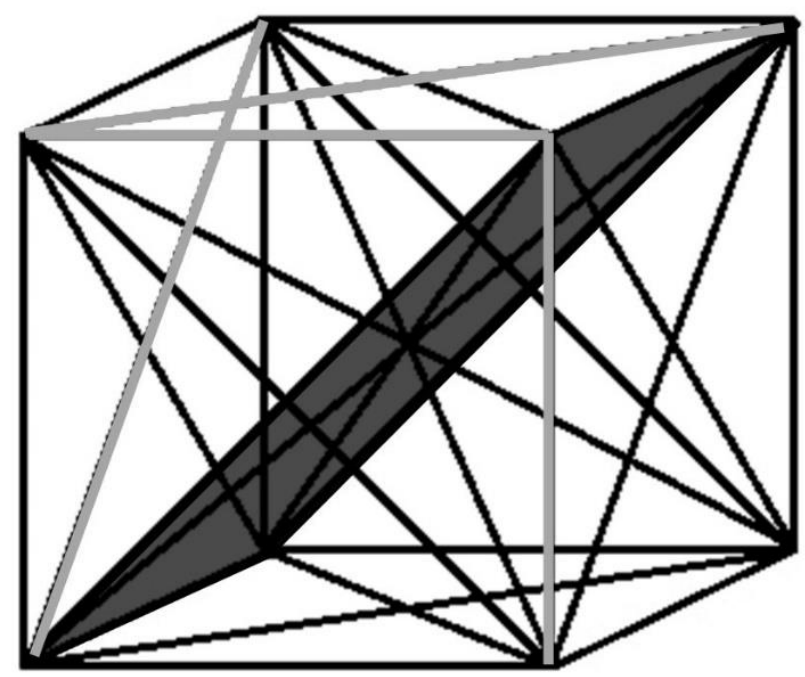

Figures 1A-B. Ramsey's theorem in case of Ramsey's number $\mathrm{R}(3,3)=6$. 1A: a complete $\mathrm{K}_{6}$ graph, geometrically forming the edge set of an exahedron with 15 edges. 1B: once connected every two vertices with a black or grey edge, RT states as follows: no matter how the black or grey lines are arranged, there will always be a triangle that is either black, or grey. In the Figure, one of the many available black triangles is depicted.

Figure 1C. Ramsey's theorem in case of $\mathrm{R}(5,5)=43$. The best coloring of the complete graph $\mathrm{K}_{43}$ contains monochromatic figures (the black pentagon in Figure) sitting on 5 nodes. Modified from: Molnár et al. (2018).

Figure 1D. Ramsey's theorem in the case of a complete three-dimensional graph with 8 edges, i.e., a hypercube. The Figure shows that this hypercube can contain a black rectangle, i.e., a monochromatic $\mathrm{K}_{4}$ with four coplanar vertices. Modified from: https://sites.google.com/site/pointlesslargenumberstuff/home/2/grahamsnumber 


\section{THE RAMSEY'S THEOREM AND THE MATHEMATICS OF THE HUMAN BRAIN CONNECTOME}

In the last decades, data containing rich relation information among elements have been widely used to perform graph theoretical analysis, such as, e.g., the building of graph neural networks, graph convolutional networks, graph recurrent networks and so on (Zhou et al., 2020). In neuroscience, the human brain connectome has been mapped through diffusion-MRI based techniques, attaining graphs equipped with up to 1015 vertices and several thousand edges (Fellner et al., 2020a). Every node usually corresponds to an anatomically labeled area or subarea of the gray matter, while every edge to the axonal fibers connecting different areas or subareas. Brain connectivity patterns (both anatomical and functional) are classified is terms of statistical dependencies (functional connectivity) or of causal interactions (effective connectivity) among various neural units, allowing neuroscientists and medical doctors to make inferences regarding human cognition, behavior and nervous disorders (Farahani et al., 2019).

Our suggestion is to use RT for the assessment of neuronal networks, in order to improve our knowledge of the connectivity among different brain subareas. Our task requires the existence in the human brain connectome of rather peculiar graphs, i.e., graphs that must be complete. Therefore, the question is: does the human brain connectome contain graphs in which every pair of distinct brain subareas is connected by a unique edge? The answer is affirmative, thanks to the works of You et al. (2020) and Fellner et al. (2020b). You et al. (2020) explored the design space of relational graphs according to their graph measures, including average path length and clustering coefficient, in which the complete graph corresponds to a fully connected layer. In turn, Fellner et al. (2020b) provided an effort to map the complete graphs of the human brain networks. Assessing the human braingraphs of 413 subjects from the dataset of the Human Connectome Project, each one consisting of 463 nodes, they listed the frequent densely connected, complete subgraphs present in at least the $80 \%$ of the available samples. Figure $\mathbf{2 A}$ provides an example of one of the $\mathrm{K}_{4}$ subgraphs detected by their graph theoretical analysis. It is noteworthy that just a few complete subgraphs display nodes in both the hemispheres. Most of these subgraphs are in the striatum, since each complete subgraph contains either a caudate nucleus, or a nucleus accumbens of either the right- or the left hemisphere. The right hippocampus and the right amygdala occur in much more complete subgraphs than the left ones; the left thalamus-proper, the left putamen and the left pallidum occur in much more complete subgraphs than the right ones.

For our RAM purposes, the required nervous subgraphs must be equipped with a number of vertices $\geq 6$. Fellner et al. (2020) found two $\mathrm{K}_{7}$ and fourty-eitght $\mathrm{K}_{6}$ complete subgraphs endowed in at least $80 \%$ of the connectomes, mostly (forty-two) located in the left hemisphere.

The two largest $\mathrm{K}_{7}$ complete subgraphs are in the left hemisphere:

a) The first one connects the left putamen with six vertices in the left frontal lobe. The seven vertices correspond to the following anatomical zones: Left-Putamen, lh.lateralorbitofrontal_4, lh.lateralorbitofrontal_6, lh.lateralorbitofrontal_7, lh.parstriangularis_3, lh.rostralmiddlefrontal_12, lh.rostralmiddlefrontal_9

b) the second one connects the left caudate and the left putamen to five left frontal areas. The seven vertices correspond to the following anatomical zones: Left-Caudate, Left-Putamen, lh.lateralorbitofrontal_7, lh.medialorbitofrontal_2, (lh.rostralanteriorcingulate_1, lh.rostralmiddlefrontal_12, lh.rostralmiddlefrontal_9

Concerning the $\mathrm{K}_{6}$ subgraphs detected in the human central nervous system, a few examples are provided in which each one of the six vertices vertex corresponds to an anatomical zone:
a) Left-Putamen,
lh.lateralorbitofrontal_6,
lh.lateralorbitofrontal_7,
lh.parstriangularis_3, lh.rostralmiddlefrontal_12, lh.rostralmiddlefrontal_9. This sugraph is illustrated in Figure 2B.
b) Left-Putamen, lh.lateralorbitofrontal_4,
lh.lateralorbitofrontal_6,
lh.parstriangularis_3, lh.rostralmiddlefrontal_12, lh.rostralmiddlefrontal_9
c) Right-Accumbens-area, Right-Caudate, Right-Hippocampus, Right-Pallidum, Right-Putamen, Right- Thalamus-Proper
d) Right-Amygdala, Right-Caudate, Right-Hippocampus, Right-Pallidum, Right-Putamen, Right-Thalamus- Proper
e) Left-Caudate, Left-Putamen, Left-Thalamus-Proper, lh.precentral_11, lh.precentral_4, lh.precentral_7
f) Left-Caudate, Left-Putamen, lh.caudalanteriorcingulate_1, lh.rostralanteriorcingulate_1, lh.rostralanteriorcingulate_2

Our first step was to demonstrate the existence in the human brain connectome of the complete subgraphs required by RT, equipped with at least six vertices. The next step is to make clear the RAM procedures to assess the neuronal networks and improve our knowledge of the brain functions. Figure $\mathbf{2 B}$ illustrates one of the $\mathrm{K}_{6}$ complete subgraphs endowed in at least $80 \%$ of the human connectomes (Fellner et al., 2020b). Every vertex stands for a different anatomical brain subarea. Every vertex is represented either as a black dot standing for an activated subarea, or a grey 
dot standing for a deactivated subarea. When an edge links two vertexes simultaneously displaying the same color, this edge is depicted with the same colour of the two vertices. In Figure 2B, for example, three subareas, being simultaneously activated, are painted like a triangle with black edges. RT suggests that in each of the forty-eight $\mathrm{K}_{6}$ complete subgraphs endowed in the human brain connectome there must always be at least three brain subareas that are simultaneously either activated or deactivated.

Apart from the opportunity to predict the simultaneous activation of brain subareas (i.e., the vertices of complete graphs) connected by neural paths (i.e., the edges of complete graphs), RT also suggests another useful application. Once detected through fMRI a few brain subareas that are simultaneously activated, the Ramsey's number provides a methodology to look for the subtending complete networks linking these brain subareas. To make an example, if the simultaneous activation of four subareas is detected in which every pair of distinct vertices is connected by a unique edge, the ensuing quadrangle must be encompassed inside a complete graph of at least 18 vertices, i.e., at least 18 brain subareas. This approach paves the way to the possibility to discover unexplored connexions among unrecognised brain subareas that are simultaneously activated (or deactivated).

In conclusion, the approach to the brain connections allowed by RAM is twofold:

1) to start from a known complete network and look for the brain subareas that must necessarily be simultaneously activated.

2) to start from the simultaneously activated brain subareas and look for the subtending, unknown complete network. 
A
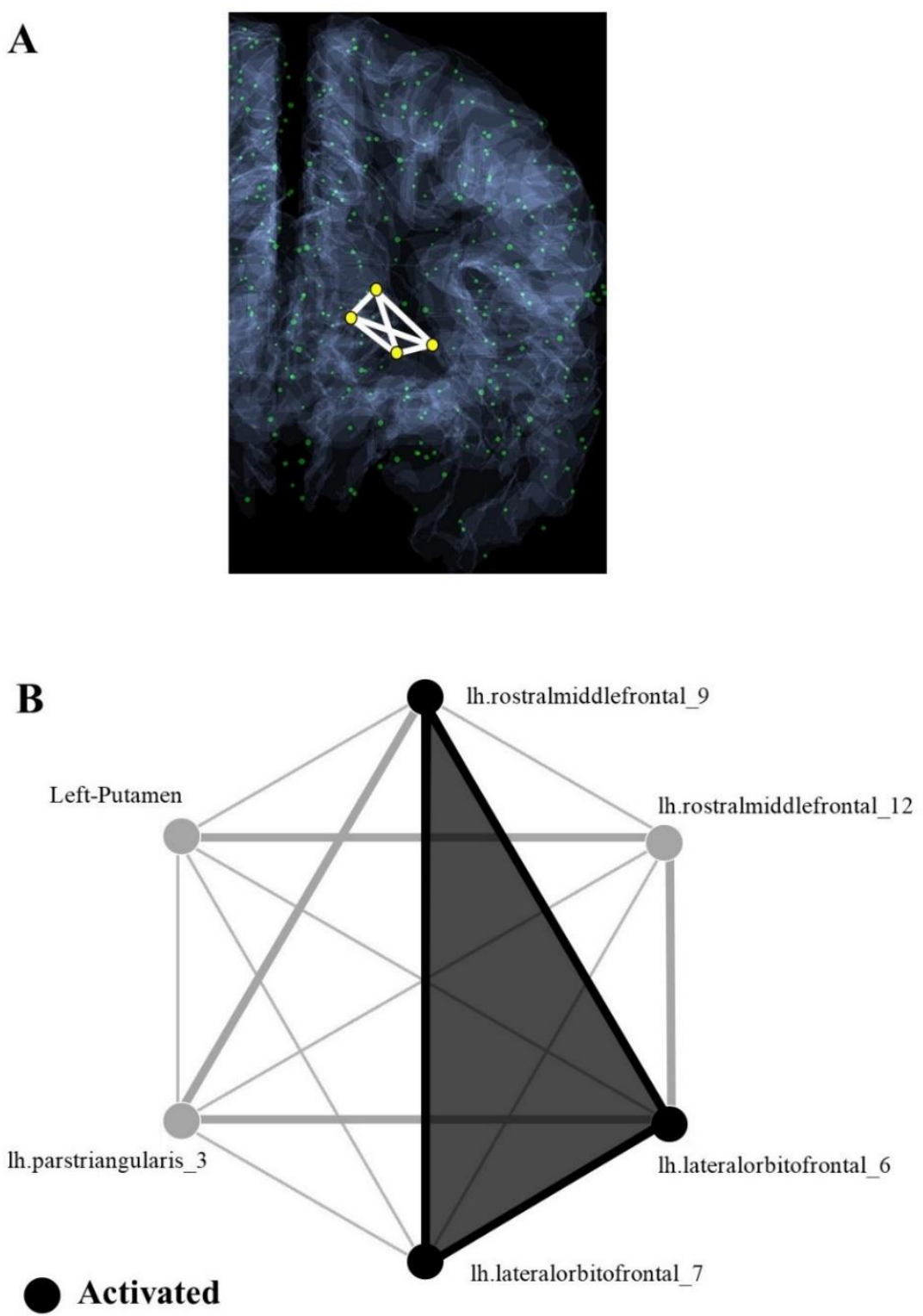

Deactivated
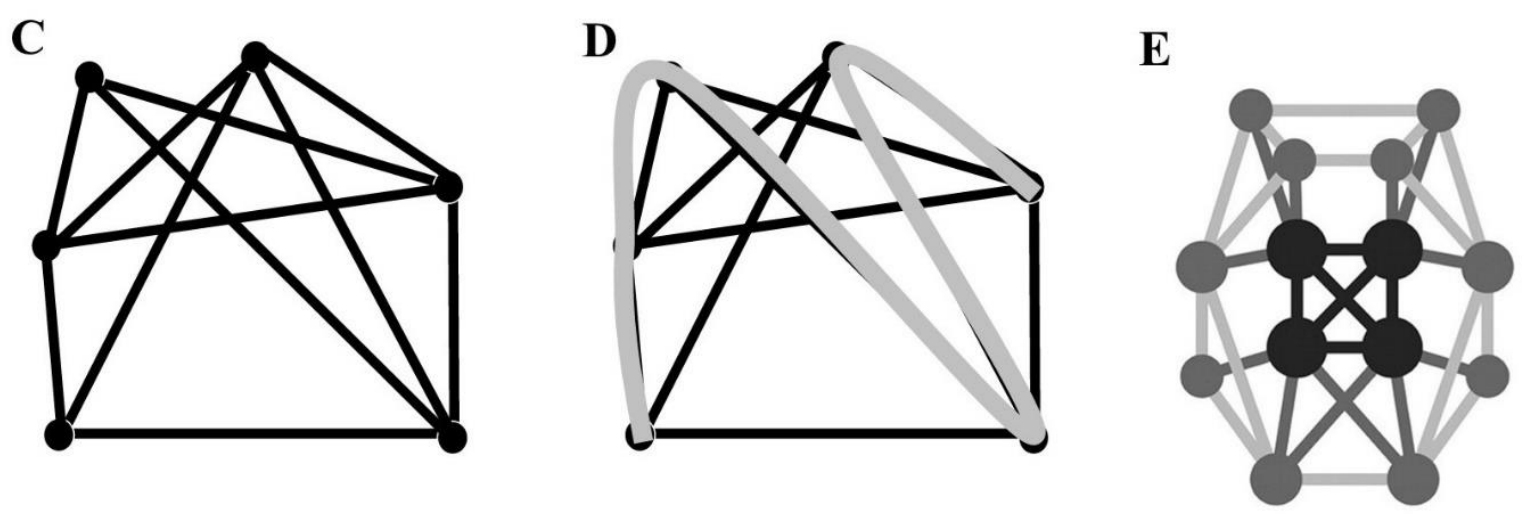

Figure 2A. A complete subgraph with four vertices endowed in the human connectome. This $\mathrm{K}_{4}$ graph connects four anatomical zones, termed: Left-Caudate, Left-Pallidum, Left-Putamen and Left-Thalamus-Proper. Modified from Fellner et al. (2020b). Figure 2B. In this $\mathrm{K}_{6}$ graph, every vertex stands for one of the anatomical subareas of the connectome described by Fellner et al. (2020b). Each vertex is illustrated as a black dot (an activated brain subarea) or 
a grey dot (a deactivated brain subarea). If an edge links two vertexes with the same colour, the edge will be of the same colour. The figure depicts the occurrence of a black triangle with black edges in which three brain subareas are simultaneously activated.

Figures 2C-E. Dirac's theorem and the human brain connectome. Given a simple graph with 6 vertices (Figure 2C), a path visiting each vertex exactly once (grey line) is allowed if all the vertices have a degree at least $n / 2$ (Figure 2D). In the case of Figure 2C, the degree is at least three. Figure 2E: in turn, the Dirac's theorem does not hold when the whole connectome is assessed as a single graph. In this simplified sketch of the bilateral human connectome (van den Heuvel and Sporns, 2011), every vertex of this 14-vertices graph does not display more than 6 edges: this means that a path that visits each vertex exactly once is forbidden by the Dirac's theorem.

\section{CONCLUSIONS}

We suggest that a Ramsey's theory-framed narrative of human brain's connectome dynamics could be able to provide alternative approaches in respect to graph theoretical analysis of neuronal networks. One of the widely used theorem from RAM is the Ramsey's theorem (RT). RT issues are generally tackled in terms of edge-coloured complete graphs, such that the theorem guarantees a monochromatic coloring in any two-edge-coloured vertex complete graph. For example, given six brain areas that form a complete graph, RT dictates that that three of them must be simultaneously activated or deactivated. Direct connections between brain subareas are of special interest, since they may contribute to enlighten the fine structure-function of the central nervous system. RT requires the existence of complete graphs endowed in the human brain connectome, such that every pair of distinct brain subareas must be connected by a unique undirected edge. These complete graphs do exist in the human brain connectome, as demonstrated by Fellner et al. (2020b). It must be emphasized that the complete graphs do not have the structure of the top-performing neural networks, since they are characterized by two detrimental features: very high clustering coefficient and very low average path length (You et al., 2020). Nevertheless, complete graphs are similar to a subgroup of real biological neural networks, in particular the ones displaying densely connected "rich club property" (van den Heuvel and Sporns 2011; Ball et al., 2014). Another invaluable advantage of complete graphs is that no anatomical contiguity among the brain subareas located in every vertex is required. To provide an example, take the vertices 1 and 2 that have been termed lh.rostralmiddlefrontal_9 and Left-Putamen in Figure 2B. The very configuration of a complete graph permits to locate the two above mentioned anatomical areas in whatsoever vertex, since in a complete graph every subarea is adjacent to every other. This means that the brain subareas which are part of a complete graph always display functional connectivity, even if they are not anatomically adjacent.

As stated above, RT can be operationally used to solve two inverse questions. On one hand, in any graph in which all points are connected by either black lines or grey lines, a large subset of the graph that is either all black or all grey is guaranteed. On the other hand, every uniform subset (no matter how it is great) is unavoidably encompassed in a graph with a well-known minimum number of vertices.

Apart from RT, RAM includes other useful theorems that could be helpful to improve the graph theoretical analysis of the human brain connectome. A subfield of RAM, i.e., the extremal graph theory (EGT), focuses on graphs with sufficiently many edges to encompass the required structures as intermediate-sized subgraphs (Bondy 1971; Szemerédi 1975; Bollobás 2004). Interesting possibilities could be given by intriguing EGT theorems, such as, for example (Motzkin and Straus, 1965; Bárány and Valtr, 1998; Büyükçolak et al., 2019):

a) Turán's theorem. An $n$-vertex complete, undirected balanced $k$-partite graph does not contain a clique with number of vertices $k_{k+1}$, but every $n$-vertex graph with more edges must contain $k_{k+1}$ clique.

b) the Erdös-Stone theorem. The number of $o\left(n^{2}\right)$ more edges guarantee the presence of any fixed subgraph of chromatic number $k+1$.

c) Erdős-Szekeres Theorem. Suppose $a, b$ in $N, n=a b+1$, and $x \_1, \ldots, x \_n$ is a sequence of $n$ real numbers. Then this sequence contains a monotonic increasing (decreasing) subsequence of a+1 terms or a monotonic decreasing (increasing) subsequence of $b+1$ terms.

d) Dirac's theorem. Every $n$-vertex simple graph all of whose vertices have degree at least $n / 2$ has a Hamilton cycle, i.e., is a path that visits each vertex exactly once.

To provide an example of the countless EGT possibilities in the study of nervous connectomics, take a simple graph (Figure 2C). Contrary to complete graphs, simple graphs are unweighted, either connected or disconnected, undirected graphs containing no graph loops or multiple edges (Bronshtein and Semendyayev 2004; Christofides et al., 2012). Since the simple graph depicted in Figure 2C displays vertices with degree at least $n / 2$, the Dirac's theorem dictates that a path crossing each vertex exactly once must unavoidably occur (Figure 2D). Therefore, the existence of 
Hamiltonian paths is mandatory inside the substructures endowed in the human brain connectome. In turn, a path that crosses each vertex exactly once is not allowed when the macro-structure of the whole connectome is assessed in terms of a single, huge simple graph. In this case, the simple graph's requirements of the Dirac's theorem are not guaranteed (Figure 2E). > Therefore, quick paths visiting just once a series of brain subareas are allowed, while quick paths visiting just once all the brain areas are not allowed. This means that a nervous path must be redundant to cross the whole brain, since it is required to cross at least a single node $\geq 2$ times.

RAM could be used also in neuroscientific disciplines different from connectomics. Take, for example, the Holland's hexagon model, widely used for the experimental evaluation of vocational interests (Holland, 1997). This model consists of a complete graph that incorporates six interrelated interest areas, each one standing for a vertex: Realistic, Investigative, Artistic, Social, Enterprising, and Conventional. A RAM approach suggests that at least three of these six features must be either present or absent in subjects undergoing MRI and voxel-based morphometry to investigate the psychometric relationships between vocational interests and abilities (see, e.g., Schroeder et al., 2012). Another feasible possibility is to correlate RT with the Boolean logic. In our example of Figure $\mathbf{2 B}$, the activation/ deactivation of brain subareas might correspond to the Boolean functions YES/NO. This means that, with the proper adjustments, RAM could be also used to assess complete graph-like Boolean networks.

By an epistemological standpoint, a RAM approach to neuronal issues suggests that the simultaneous activation of brain subareas (usually detected through pairwise comparison techniques) subtending brain connectome dynamics might stand for a nervous mechanism depending on the intrinsic mathematical features of the graph arrangement. The flows through the edges of neural graphs in the central nervous system might require just a peculiar, inherent networks conformation, rather than specifically committed physical/biological mechanisms.

\section{DECLARATION OF INTEREST}

The author warrants that the article is original, does not infringe on any copyright or other proprietary right of any third part, is not under consideration by another journal, and has not been previously published.

The Author does not have any known or potential conflict of interest including any financial, personal or other relationships with other people or organizations within three years of beginning the submitted work that could inappropriately influence, or be perceived to influence, their work.

This research did not receive any specific grant from funding agencies in the public, commercial, or not-for-profit sectors.

This research does not contain any studies with human participants or animals performed by the Author.

The Author had full access to all the data in the study and take responsibility for the integrity of the data and the accuracy of the data analysis. The Author performed: study concept and design, acquisition of data, analysis and interpretation of data, drafting of the manuscript, critical revision of the manuscript for important intellectual content, statistical analysis, obtained funding, administrative, technical, and material support, study supervision.

\section{REFERENCES}

1) Ajtai M, Komlós J, Szemerédi E. 1980. A note on Ramsey numbers. J Combin Theory Ser. A, 29 (3): $354-$ 360, doi:10.1016/0097-3165(80)90030-8.

2) Allen P. 2008. Covering Two-Edge-Coloured Complete Graphs with Two Disjoint Monochromatic Cycles. Combinatorics, Probability \& Computing, 17(4): 471 - 486.

3) Angeltveit V, McKay BD. 2017. R(5,5) $\leq 48$. arXiv:1703.08768.

4) Ball G, Aljabar P, Zebari S, Tusor N, Arichi T, Merchant N, et al. 2014. Rich-club organization of the newborn human brain. Proc Natl Acad Sci U S A. 111(20):7456-7461. http://dx.doi.org/10.1073/pnas.1324118111.

5) Bárány I, Valtr P. 1998. A Positive Fraction Erdos - Szekeres Theorem. Discrete Comput Geom 19, 335 -342. https://doi.org/10.1007/PL00009350.

6) Bollobás B. 2004. Extremal Graph Theory, New York: Dover Publications, ISBN 978-0-486-43596-1.

7) Bondy JA. 1971. Pancyclic graphs I. Journal of Combinatorial Theory, Series B, 11(1):80-84.

8) Bondy JA, Murty USR. 2008. Graph Theory. Springer Verlag; 3rd printing 2008 ed. ISBN-13: 9781846289699 . 
9) Bronshtein IN, Semendyayev KA. 2004. Handbook of Mathematics, 4th ed. New York: Springer-Verlag.

10) Büyükçolak J, Gözüpek D, Özkan S, Shalom M. 2019. On one extension of Dirac's theorem on Hamiltonicity. Discrete Applied Mathematics. Volume 252, Pages 10-16. https://doi.org/10.1016/j.dam.2017.01.011.

11) Christofides D, Kühn D, Osthus D. 2012. Edge-disjoint Hamilton cycles in graphs. Journal of Combinatorial Theory, Series B, 102(5): 1035-1060.

12) Diestel R. 2018. Graph Theory. Springer; Vth ed. ISBN-13: 978-3662575604.

13) Farahani FV, Karwowski W, Lighthall NR. 2019. Application of Graph Theory for Identifying Connectivity Patterns in Human Brain Networks: A Systematic Review. Front. Neurosci. https://doi.org/10.3389/fnins.2019.00585.

14) Fellner M, Varga B, Grolmusz V. 2020a. The frequent network neighborhood mapping of the human hippocampus shows much more frequent neighbor sets in males than in females. PLOS ONE. 2020; 15(1):e0227910. https://doi.org/10.1371/journal.pone.0227910.

15) Fellner M, Varga B, Grolmusz V. 2020b. The frequent complete subgraphs in the human connectome. PLoS One, 15(8):e0236883. doi: 10.1371/journal.pone.0236883.

16) Graham RL, Rothschild BL. 1971. Ramsey's Theorem for n-Parameter Sets. Transactions of the American Mathematical Society. 159: 257-292. doi:10.2307/1996010.

17) Graham RL, Rothschild BL, Spencer JH. 1990. Ramsey Theory, 2nd ed. New York: Wiley.

18) Hindman N, Strauss D. 1998. 18 Multidimensional Ramsey Theory. In: Hindman N, Strauss D. Algebra in the Stone-Cech Compactification Theory and Applications. Volume 27 in the series De Gruyter Expositions in Mathematics. https://doi.org/10.1515/9783110809220.369.

19) Holland JL: Making Vocational Choices: A Theory of Vocational Personalities and Work Environments. 1997, Psychological Assessment Resources, Inc, Lutz, FL, 3

20) Matelsky, J.K., Reilly, E.P., Johnson, E.C. et al. 2021. DotMotif: an open-source tool for connectome subgraph isomorphism search and graph queries. Sci Rep 11, 13045. https://doi.org/10.1038/s41598-02191025-5.

21) Molnár B, Varga M, Toroczkai Z, Ercsey-Ravasz M. 2018. A high-performance analog Max-SAT solver and its application to Ramsey numbers. arXiv:1801.06620

22) Motzkin TS, Straus EG. 1965. Maxima for graphs and a new proof of a theorem of Turán. Canadian Journal of Mathematics, 17: 533-540, doi:10.4153/CJM-1965-053-6.

23) Peters JF, Tozzi A, Deli E. 2017. Towards Equations for Brain Dynamics and the Concept of Extended Connectome. SF J Neuro Sci 1:1.

24) Reese TM, Brzoska A, Yott DT, Kelleher DJ. 2012 Analyzing Self-Similar and Fractal Properties of the C. elegans Neural Network. PLoS ONE 7,(10): e40483. (doi:10.1371/journal.pone.0040483).

25) Roberts FS. 1984. Applications of Ramsey theory. Discrete Applied Mathematics. Volume 9(3):251-261.

26) Rosta V. 2004. Ramsey Theory Applications. Electronic J Combinatorics, DOI: https://doi.org/10.37236/34.

27) Schroeder DH, Haier RJ, Tang CY. 2012. Regional gray matter correlates of vocational interests. BMC Res Notes 5, 242. https://doi.org/10.1186/1756-0500-5-242.

28) Szemerédi E. 1975. On sets of integers containing no k elements in arithmetic progression. Acta Arith, 27 (585):199-245.

29) Sporns O, Tononi G, Kötter R. 2005. The human connectome: A structural description of the human brain. PLoS Computational Biology. 1 (4): e42. doi:10.1371/journal.pcbi.0010042.

30) You J, Leskovec J, He K, Xie S. 2020. Graph Structure of Neural Networks. arXiv:2007.06559.

31) van den Heuvel MP, Sporns O. 2011. Rich-Club Organization of the Human Connectome. Journal of Neuroscience, 31 (44) 15775-15786; DOI: https://doi.org/10.1523/JNEUROSCI.3539-11.2011.

32) Wilson RJ. 2010. Introduction to Graph Theory. Prentice Hall; 5th Ed. ISBN-13 : 978-0273728894.

33) Zhou J, Cui G, Hu S, Zhang Z, Yang C, et al. 2020 Graph neural networks: A review of methods and applications. AI Open, Volume 1, 2020, Pages 57-81. 